\title{
Contrast Induced by a Static Magnetic Field for Improved Detection in Nanodiamond Fluorescence Microscopy
}

\author{
Shashi K. R. Singam, ${ }^{1}$ Jaroslaw Motylewski, ${ }^{2}$ Antonina Monaco, ${ }^{2}$ Elena Gjorgievska, ${ }^{3}$ Emilie Bourgeois, ${ }^{3}$ \\ Milos Nesládek, ${ }^{3}$ Michele Giugliano, ${ }^{2}$ and Etienne Goovaerts, ${ }^{1, *}$ \\ ${ }^{1}$ Physics Department University of Antwerp, Universiteitsplein 1, BE-2610 Antwerpen, Belgium \\ ${ }^{2}$ Theoretical Neurobiology and Neuroengineering Laboratory, Department of Biomedical Sciences, \\ University of Antwerp, Universiteitsplein 1, BE-2610 Antwerpen, Belgium \\ ${ }^{3}$ Institute for Materials Research (IMO), University Hasselt Wetenschapspark 1, \\ B-3590 Diepenbeek, Belgium
}

(Received 17 May 2016; revised manuscript received 25 October 2016; published 27 December 2016)

\begin{abstract}
Diamond nanoparticles with negatively charged nitrogen-vacancy (NV) centers are highly efficient nonblinking emitters that exhibit spin-dependent intensity. An attractive application of these emitters is background-free fluorescence microscopy exploiting the fluorescence quenching induced either by resonant microwaves (RMWs) or by an applied static magnetic field (SMF). Here, we compare RMWand SMF-induced contrast measurements over a wide range of optical excitation rates for fluorescent nanodiamonds (FNDs) and for NV centers shallowly buried under the (100)-oriented surface of a diamond single crystal (SC). Contrast levels are found to be systematically lower in the FNDs than in the SC. At low excitation rates, the RMW contrast initially rises to a maximum (up to 7\% in FNDs and 13\% in the SC) but then decreases steadily at higher intensities. Conversely, the SMF contrast increases from approximately $12 \%$ at low excitation rates to high values of $20 \%$ and $38 \%$ for the FNDs and SC, respectively. These observations are well described in a rate-equations model for the charged NV defect using parameters in good agreement with the literature. The SMF approach yields higher induced contrast in image collection under commonly applied optical excitation. Unlike the RMW method, there is no thermal load exerted on the aqueous media in biological samples in the SMF approach. We demonstrate imaging by SMF-induced contrast in neuronal cultures incorporating FNDs (i) in a setup for patch-clamp experiments in parallel with differential-interference-contrast microscopy, (ii) after a commonly used staining procedure as an illustration of the high selectivity against background fluorescence, and (iii) in a confocal fluorescence microscope in combination with bright-field microscopy.
\end{abstract}

DOI: 10.1103/PhysRevApplied.6.064013

\section{INTRODUCTION}

Because of their particular photophysical properties, the nitrogen-vacancy (NV) centers incorporated in diamond nanoparticles have recently become the basis for successful nanoscale sensing in biomedical sciences [1-5]. In particular, they have been introduced in fluorescence microscopy because of the high quantum yield of the NV fluorescence emitted in a favorable spectral window for biological applications. These advantages come together with the stability and chemical inertness of the diamond matrix. In recent years, there have been several reports of in vivo imaging in far-field and confocal microscopy based on fluorescent nanodiamond (FND) particles [1,6-8]. Motivated by this, important efforts are being made to improve their efficiency, biocompatibility, and biofunctionality [8-12]. The negatively charged NV defect possesses a spin-triplet electronic ground state [13] and a system of

* Corresponding author.

Etienne.Goovaerts@uantwerpen.be triplet and singlet excited states [14-17] that imparts unique spin-dependent properties that can be exploited in a multitude of applications such as magnetometry [18-20], magnetic imaging [21-23], local ionic potential [24,25], thermometry [26,27], and detection of magnetic fluctuations $[28,29]$ at the nanoscale $[3,30]$, as well as single-photon emitters [31] and qubits for quantum computing and cryptography $[32,33]$. An application of high interest is magnetometry to detect neuronal action potentials; its feasibility was first discussed several years ago [34], and very recently, a breakthrough demonstration was reported [35].

A few different approaches have been reported for selective detection of FND emission with respect to autofluorescence or other background signals. Fluorescencetime-imaging microscopy (FLIM) is an elegant method based on the longer lifetime of NV fluorescence relative to that of typical emission in biological samples (approximately $20 \mathrm{~ns}$ vs approximately $3 \mathrm{~ns}$ ) [5,6,36]. The latter background is efficiently eliminated and does not directly contribute to the shot noise. However, the gating significantly decreases the collection of photons which indirectly reduces 
(a)

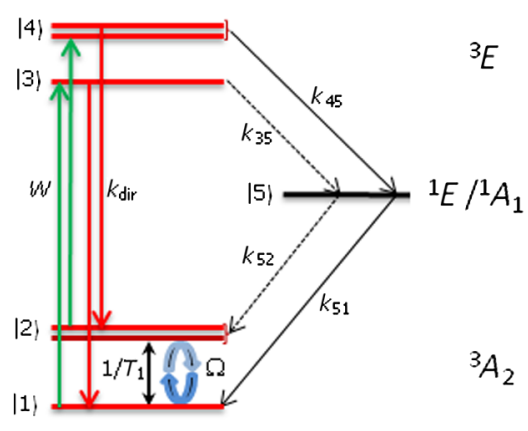

(b)

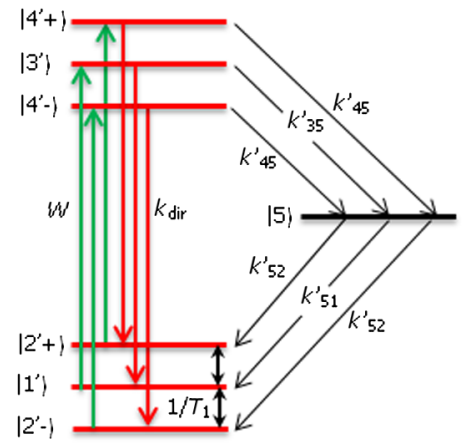

(c)

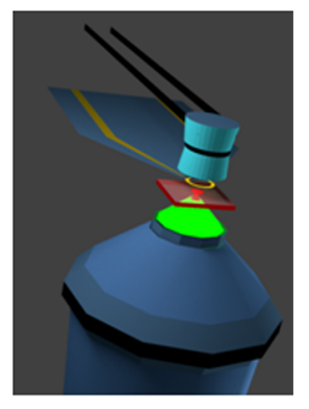

FIG. 1. Energy-level schemes of the $\mathrm{NV}^{-}$center in diamond [42] in (a) zero field and (b) a strong static magnetic field. The relevant parameters for spontaneous, optical, and microwave transitions are indicated. (c) Schematics picture of the experimental setup showing the sample, the microwave antenna loop, and the permanent magnet (sideways removable) in front of the microscope objective. the SNR. FLIM further requires a relatively complex combination of pulsed laser excitation and fast-gating detection. This imposes serial detection of the image pixels which limits the range of applications in biological microscopy. Alternatively, the spin dependence of the emission intensity of the $\mathrm{NV}^{-}$defect has been exploited for background-free NV fluorescence microscopy. External control over the spin polarization is obtained either by resonant microwave (RMW) excitation [37,38], by application of a static magnetic field (SMF) [39,40], or a combination of both that also offers 3D-imaging capabilities [41]. These methods have been demonstrated for background-free microscopy in animal tissue [41], microorganisms [37,38], and small animals $[37,40]$. These spin-based methods allow for important improvements in terms of selectivity of detection, SNR, penetration depth in tissues, etc. It is, therefore, important to understand the optimal conditions of application for the proposed approaches.

The $\mathrm{NV}^{-}$defect with $C_{3 v}$ symmetry is an efficient emitter in the (630-800)-nm region when optically excited from the ground ${ }^{3} A_{2}$ to the excited ${ }^{3} E$ electronic states [see Fig. 1(a)] $[15,16]$. Optical excitation in the green region excites the $\mathrm{NV}^{-}$defect in vibrationally excited levels of the ${ }^{3} E$ states. The magnetic sublevels of the ground state are in zero magnetic field split by $2.87 \mathrm{GHz}$ between a lowest nondegenerate state $\left(m_{S}=0\right.$, level 1) and a doubly degenerate level $\left(m_{S}= \pm 1\right.$, level 2$)$ consisting of spin states quantized along the $\langle 111\rangle$ trigonal symmetry axis. Optical transitions are spin preserving between the ${ }^{3} A_{2}$ levels 1 and 2 and the ${ }^{3} E$ levels 3 and 4, respectively, and rates for excitation $\left(W=W_{13}=W_{24}\right)$ and direct decay $\left(k_{\text {dir }}=k_{31}=k_{42}\right)$ can be assumed for these two allowed transitions. From excited level 4, and to a lesser extent from level 3, competitive nonradiative decay (rates $k_{45}>k_{35}$ ) can occur via intersystem crossing (ISC) towards a system of intermediate singlet states (represented here by level 5), lowering the quantum yields for emission from the ${ }^{3} E$ states. Moreover, the lowest intermediate ${ }^{1} E$ singlet [17] is decaying preferentially towards level 1 in the ${ }^{3} A_{2}$ ground state $\left(k_{51}>k_{52}\right)$, which together with the marked selectivity of the ISC from ${ }^{3} E\left(k_{45}>k_{35}\right)$ is at the origin of pronounced spin polarization towards level 1 in the ground state under optical excitation. As this level possesses the higher quantum yield for emission, this spin polarization enhances the overall fluorescence efficiency. Table I lists a set of dynamical rate parameters for the $\mathrm{NV}^{-}$defect collected from the recent literature. One notices the slow relaxation rate for the ground-state spin polarization corresponding to a long population lifetime $T_{1}$ of several milliseconds at room temperature [44], which is a key element to explain the significant levels of spin polarization that can be reached.

The RMW contrast exploited for background-free microscopy is based on microwave-induced spin transitions (Rabi frequency: $\Omega$, proportional to $B_{1}$, the transverse microwave field component) tending to equalize the populations of the sublevels of the ground state and, thus, to neutralize the increase in fluorescence obtained from spin polarization [37]. The origin of the SMF contrast is quite different: quantization of the spin states is now imposed along the static magnetic field that is splitting level 2 and mixing the original spin states $[39,45]$. This quantization in a sense scrambles the ISC transitions leading to modified values of the corresponding

TABLE I. Literature values for the rate parameters in the fivelevel model and our selection of values for the calculated results discussed below (see Figs. 4 and 5).

\begin{tabular}{lcc}
\hline \hline Parameter & $\begin{array}{c}\text { Literature } \\
\text { values }^{\mathrm{a}}\left(\mathrm{s}^{-1}\right)\end{array}$ & $\begin{array}{c}\text { Values used in } \\
\text { simulation }\left(\mathrm{s}^{-1}\right)\end{array}$ \\
\hline$k_{\mathrm{dir}}=k_{31}=k_{42}$ & $(66 \pm 5) \times 10^{6}$ & $66 \times 10^{6}$ \\
$k_{35}$ & $(7.9 \pm 4.1) \times 10^{6}$ & $11 \times 10^{6}$ \\
$k_{45}$ & $(53 \pm 7) \times 10^{6}$ & $53 \times 10^{6}$ \\
$k_{51}$ & $(1.0 \pm 0.8) \times 10^{6}$ & $1.0 \times 10^{6}$ \\
$k_{52}$ & $(0.35 \pm 0.25) \times 10^{6}$ & $0.60 \times 10^{6}$ \\
$T_{1}^{-1}$ & $160-350$ & 210 \\
$T_{2}^{-1}$ & $\sim 10^{6}$ & $0.65 \times 10^{6}$ \\
\hline \hline
\end{tabular}

${ }^{\mathrm{a}}$ Transition rates $k_{i j}$ from Table II in Ref. [18] based on Ref. [43]. Note the different indexing of the states and the different definition of the $k_{52}$ parameter (see Appendix A), which is half of the parameter $k_{62}$ as used in Dumeige et al. [18]. Value of $T_{1}{ }^{-1}$ from Ref. [44] (variation among several single $\mathrm{NV}^{-}$ centers in SC diamond). 
rates [see Fig. 1(b)], which are strongly dependent on the angle between the field and trigonal axis (see, e.g., Ref. [43]). The resulting spin polarization decreases when the field is turned away from the defect axis and, in particular, vanishes at the magic angle. The spin polarization and the resulting contrast are dynamically induced by the optical excitation and will, therefore, depend on the intensity of the latter. As described by Dréau et al. [46], increasing the optical excitation decreases the RMW contrast which tends to vanish above a characteristic level. Increasing the microwave excitation to counter this effect is, in practice, severely limited by heating effects that can easily cause damage to biological water-containing samples.

Here we report the parallel measurements of RMW- and SMF-induced contrast in $\mathrm{NV}^{-}$fluorescence over a wide range of optical excitation rates. They are performed on FNDs as well as on $\mathrm{NV}^{-}$defects incorporated in a diamond epitaxial layer grown on a (100) surface of a diamond single crystal (SC), the latter providing a model system with high homogeneity and well-defined defect orientations. Contrary to the RMW method, the SMF approach yields improving contrast with increasing intensity of the optical excitation for FNDs as well as in the SC. We present a quantitative analysis of the data using a rate-equation model based on the energy-level scheme presented in Fig. 1, closely related to the models applied in previous reports $[18,43,47]$. This model also explains the intensitydependent behavior in the FNDs. From our results, the SMF contrast is shown to be the method of choice for imaging of localization and distribution of FNDs, which provides a general-purpose microscopy tool for ongoing research on biological applications of functionalized FNDs targeting specific molecules and cell constituents. As a proof of concept, we present SMF-contrast imaging of FNDs incubated with primary neuronal cultures combined with differential-interference-contrast (DIC) imaging of the cells performed in a microscope for standard patch-clamp experiments and showing the colocalization with cell structures. Moreover, we demonstrate very efficient discrimination between nanodiamond fluorescence and background emission for neuronal cultures treated to selectively reveal neuronal cells using beta-Tubulin III antibodies. Finally, an extension of the approach to 3D imaging is illustrated by confocal fluorescence microscopy with parallel bright-field imaging in a FND-seeded neuronal culture.

\section{EXPERIMENT}

The single-crystal diamond layers used in the intensitydependent contrast measurements are grown by plasmaenhanced chemical vapor deposition (PE CVD) using an ASTeX PDS17 microwave PE CVD reactor. The details for the deposition of epitaxial layers can be found in Ref. [48]. The epitaxial 32.8- $\mu \mathrm{m}$-thick film is grown at a rate of $6 \mu \mathrm{m} / \mathrm{h}$ on a $\left(3 \times 3 \times 0.3 \mathrm{~mm}^{3}\right)$ commercial highpressure high-temperature substrate (Element Six, UK) with a (110) off-axis polish $2.3^{\circ}$ from the (100) plane. The growth is carried out in a $\mathrm{H}_{2} / \mathrm{CH}_{4}$ mixture containing $1 \% \mathrm{CH}_{4}$, at a total flow of $500 \mathrm{sccm}$, pressure 88 torr, and substrate temperature $810^{\circ} \mathrm{C}$. Gas-phase $\mathrm{N}$ doping with $\mathrm{N}_{2}$ is applied at a concentration of $2000 \mathrm{ppm}(\mathrm{N}: \mathrm{C}$ ratio). The $\mathrm{PL}$ of the film exhibits a typical spectrum of a NV center predominantly from $\mathrm{NV}^{-}$centers, with a contribution of $\mathrm{NV}^{0}$ at $650 \mathrm{~nm}$ less than $10 \%$ of the total PL signal. From confocal fluorescence microscopy performed in an optimized home-built setup for NV color center detection [24], the $\mathrm{NV}^{-}$concentration in the produced epilayer is estimated to be approximately $600 \mathrm{ppb}$ calibrated against CVD diamond with known $\mathrm{NV}^{-}$center concentration. (Further data of the epilayer growth and the $\mathrm{NV}^{-}$center distribution are provided in the Supplemental Material [49].) The SC is positioned on a glass coverslip during the measurements in an inverted microscope setup.

The intensity-dependent contrast measurements in FNDs are performed on nanodiamonds fabricated starting from a commercial powder with particle size of 35-40 nm from MikroDiamant as detailed in Ref. [50]. After an oxidizing bath, the solution is lyophilized and further irradiated with $16-\mathrm{MeV}$ protons (fluency: $5.0 \times 10^{16} \mathrm{H}^{+} / \mathrm{cm}^{2}$ ) at the Institute of Nuclear Physics, Czech Academy of Sciences by using the nanodiamonds compressed in a pellet target. The samples are further annealed at $850^{\circ} \mathrm{C}$ for $120 \mathrm{~min}$ to produce $\mathrm{NV}$ centers by diffusing the irradiation-produced vacancies. Typically, two to three NV centers are produced per nanoparticle [51]. Nondiamond carbon impurities resulting from irradiation and thermal annealing are removed by oxidation in air at $510^{\circ} \mathrm{C}$ for $6 \mathrm{~h}$. Dominant chemical functions on the surface of these FNDs are carboxyl and primary hydroxyl groups, as determined by XPS. Colloidal suspension is prepared from irradiated, annealed, and oxidized diamond. The $Z$ potential is $35 \mathrm{mV}$, and the size distribution shows nonclustered particles with a main size distribution of approximately $40 \mathrm{~nm}$ [51].

Alternatively, for the fluorescence-microscopy experiments, the neuronal cultures are treated with a FND-containing solution purchased from Adamas Nanotechnologies, USA, as we describe in more detail below.

The home-built inverted microscope setup is based on an Olympus tube equipped with a $40 \times$ water-immersion objective lens (Olympus, UAPON4XW340, N.A. 1.15). Images are recorded from an intensified charge-coupleddevice camera (Stanford Computer Optics, Inc., 4 Quik E camera, $752 \times 582$ ). In the same setup the sample can be excited by means of a frequency-doubled Nd:YAG laser (532 nm, Changchun New Industries Optoelectronics Tech. Co., Ltd., MLL-III-532-200 mW). Also, the emitted light can be collected either for spectral analysis using a compact spectrometer (Maya2000 Pro, Ocean Optics) or for detection of the overall intensity in the $\mathrm{NV}^{-}$emission region using an avalanche photodiode (Hamamatsu C5460-01). 
A set of long-wavelength pass filters allows passing the neutral and negatively charged NV emissions (from $570 \mathrm{~nm}$ ) or selectively only the latter [from $630 \mathrm{~nm}$, for optically detected magnetic resonance (ODMR) and background-free microscopy]. Alternatively, optical excitation can be performed in this microscope with a 525-nm power lightemitting diode (LED) (Roithner LaserTechnik, GmbH, SMB1N-525V, $1 \mathrm{~mW}$ measured at sample position) combined with a short-wavelength pass filter for purification of the excitation spectrum in the detection spectral range.

Optical-power-dependent measurements in the inverted microscope setup are acquired by variable attenuation of the laser beam calibrated by means of a power meter (Spectra Physics, model 404). At higher excitation levels, this is combined with an appropriate attenuation in the collecting light path to avoid saturation of the detector. Magnetic field and microwave acquisitions are performed in an alternating sequence to maintain similar conditions in both cases at a given excitation intensity. Reference spectra are measured at the start and end of the sequence at each optical power to check the stability of the signal.

A broadband antenna suitable for a wide frequency range around $2.87 \mathrm{GHz}$ emits the microwaves. It consists of a loop of $1 \mathrm{~mm}$ diameter defined on an electronic board in series with a $50-\Omega$ load. In these experiments, the antenna is positioned behind the sample; it is well centered and close enough for maximum impinging power [see Fig. 1(c)]. A signal generator (Rhode \& Schwarz, SMC100A) combined with a microwave amplifier (WanTcom, Inc., WBPA2736A, 24-dB amplification) produces the microwaves with up to $33-\mathrm{dBm}$ output power.

We measure ODMR spectra by direct fluorescence detection or by lock-in detection (EG\&G, Princeton Applied Research, model 5204) under on/off amplitude modulation of the microwaves (see the Supplemental Material [49]). The time dependence of the $\mathrm{NV}^{-}$fluorescence is measured under on/off microwave modulation using a storage oscilloscope Tektronix (TBS1022) to evaluate the spin-population relaxation time of the $\mathrm{NV}^{-}$ centers (see the Supplemental Material [49]).

A static magnetic field is applied from behind the antenna parallel to the optical axis (perpendicular to the sample surface) piling up two permanent magnets of $10 \mathrm{~mm}$ diameter and $4 \mathrm{~mm}$ height (E654, e-Magnets, UK). These magnets are mounted onto a moveable arm allowing for repeatable insertion or removal for measurements with and without SMF, respectively. In the inverted microscope, the magnet is positioned as close as possible, providing a field of approximately $220 \mathrm{mT}$ on the sample. This ensures that the high-field approximation is valid for the analysis of these data (see Sec. III C).

Similar provisions are made for imaging with and without magnetic field (SMF contrast) in the patch-clamp and confocal microscopes (see the description below). In these setups, due to space constraints, the typical distance for imaging experiments is $7 \mathrm{~mm}$ corresponding to a field $>50 \mathrm{mT}$. This configuration in practice yields near saturation of the contrast as a function of field (see the Supplemental Material [49]).

Field-dependent measurements (Supplemental Material [49]) are performed by variation of the distance from the sample using a Hall Gaussmeter (RFL Electronics Inc., USA) for calibration. The patch-clamp setup is equipped with an upright microscope (Leica, DM LFS) with long working distance, water-immersion objectives either of $63 \times(\mathrm{HCX}$ APO-L63 $\times / 0.3 \mathrm{WU}-\mathrm{V}-\mathrm{I})$ or $10 \times($ HCX APO-L10 $\times / 0.3 \mathrm{WU}-\mathrm{V}-\mathrm{I})$ magnification, CCD camera (PCO AG, pixelfly VGA, $640 \times 480$ ), and a standard near-infrared DIC imaging implementation in transmission. Excitation by a green laser pointer $(532 \mathrm{~nm}$, $2 \mathrm{~mW}$ on sample, Nd:YAG based) is implemented through the top illumination port of the microscope using an appropriate lens for defocusing of the beam to obtain an illuminated central area in the object plane. A customordered cube allows for fluorescence microscopy exciting at $532 \mathrm{~nm}$ and collecting in the long-wavelength range from $580 \mathrm{~nm}$. Glass coverslips are kept under a continuous flow of extracellular solution containing (in $\mathrm{mM}$ ): $145 \mathrm{NaCl}$, $4 \mathrm{KCl}, 2 \mathrm{Na}$-pyruvate, $5 \mathrm{HEPES}, 5$ glucose, $2 \mathrm{CaCl} 2$, and 1 $\mathrm{MgCl} 2 ; p \mathrm{H}$ adjusted to 7.4 with $\mathrm{NaOH}$.

Confocal imaging is performed in an upright microscopy (Olympus, model BX61WI) utilizing the spinning disk technology (Andor, Revolution DSD2) and equipped with a sCMOS CCD camera (Andor, ZYLA 5.5 USB3-500). Bright-field images in transmission are measured in parallel with confocal fluorescence microscopy, which are obtained under green excitation from a metal halide source while using standard detection filtering for red fluorescent protein.

In order to improve neuronal adhesion, we treat the surface of all glass coverslips with polyethyleneimine (PEI, Sigma-Aldrich, Belgium) prior to cell seeding [52]. The coverslips are soaked overnight in $0.1 \%$ PEI solution, then rinsed with deionized water and left drying at room temperature. Primary neuronal cultures are obtained by standard methods [53] from postnatal (day 0) Wistar rats (Charles River, France) following national and institutional guidelines on animal experimentation. Briefly, cortical brain tissue slices are sliced into small pieces and digested in $0.025 \%$ trypsin solution, followed by mechanical dissociation through the opening of a glass pipette. Before seeding, the cells are centrifuged and suspended in modified essential medium supplemented with $50-\mu \mathrm{g} / \mathrm{mL}$ gentamicin, 2-M glucose, $5 \%$ horse serum, and 200-mM L-glutamine (Sigma-Aldrich, Belgium). The cells are plated at a seeding density of 1000 cells $/ \mathrm{mm}^{2}$. After $24 \mathrm{~h}$, a solution containing FNDs (Adamas Nanotechnologies, USA, ND-15NV-40 nm, average particle size $40 \mathrm{~nm}$, carboxylate-ended surface, $Z$ potential $\zeta=-40 \mathrm{mV}$, and estimated $15 \mathrm{NV}^{-}$centers per particle) is 
(a) SC fluorescence

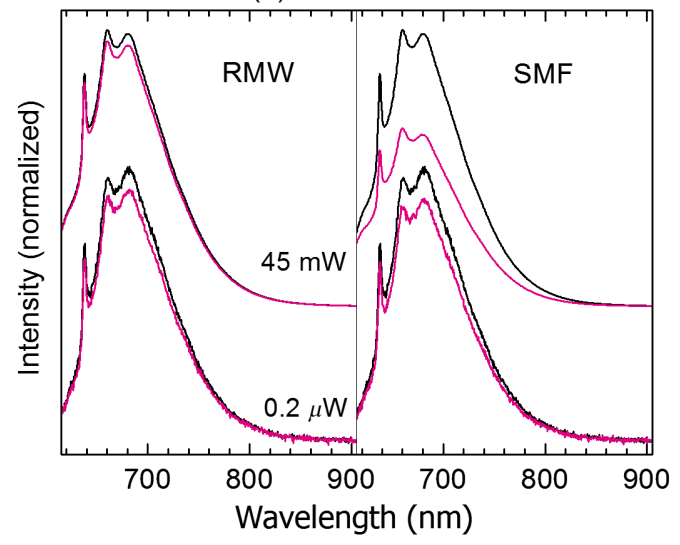

(b) FND fluorescence

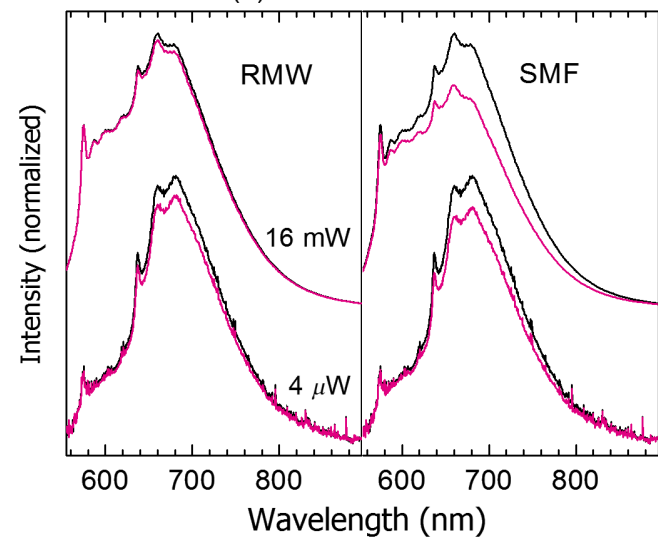

FIG. 2. Fluorescence spectra of (a) shallowly buried $\mathrm{NV}^{-}$defects in a (100) SC and (b) FNDs measured under high (upper graphs) and low (lower graphs) laser excitation intensities. The effects of RMW (left panes, 33-dBm microwave power) and SMF (right panes) perturbation are compared showing the spectra without (black) and with (colored) perturbation applied. To show the relative induced decrease, the fluorescence intensities are each time normalized to the maximum of the unperturbed spectra. added to the culture medium at a concentration of $50 \mu \mathrm{g} / \mathrm{mL}$. To minimize aggregation of diamond nanoparticles, the FND solution is vortexed for $1 \mathrm{~min}$ and then sonicated for 5 min using a 2-mm titanium probe (UP200S and S26d2, Hielscher, Germany) before being added to the medium.

Cultures are maintained for several days at $37^{\circ} \mathrm{C}, 5 \%$ $\mathrm{CO}_{2}$, and $100 \%$ humidity, and the culture medium without any further addition of FNDs is replaced three times per week. Cultured cells are immunolabeled, which is staining the neuronal cytoskeleton after 5-8 days in vitro. Briefly, samples are washed three times with phosphate-buffered saline (PBS) (Sigma-Aldrich, Belgium) at room temperature and fixed at $37^{\circ} \mathrm{C}$ with $4 \%$ paraformaldehyde for $15 \mathrm{~min}$. The cultures are subsequently washed twice with PBS before incubation for 10 min with PBS-1\% Triton (Sigma-Aldrich, Belgium). After that, the reaction is blocked for 30 min with PBS-1\% Triton supplemented with $10 \%$ goat serum. Then the samples are incubated overnight on a rocking platform at $4{ }^{\circ} \mathrm{C}$ with mouse primary monoclonal antibody antitubulin, beta III isoform (Merck Millipore, Germany). The following day, the cultures are washed three times with PBS at room temperature and incubated for $1 \mathrm{~h}$ on a rocking platform in the dark with goat antimouse secondary antibody IgG. The cultures are then washed three times with PBS and mounted with DAPI (4',6-diamidino-2-phenylindole) hardset medium and left to dry for $1 \mathrm{~h}$ at room temperature.

\section{RESULTS AND DISCUSSION}

\section{A. Intensity-dependent contrast}

The measurements of RMW- and SMF-induced contrast are performed in an inverted microscope setup with a $40 \times$ water-immersion objective as schematically shown in Fig. 1(c). An antenna loop close to the sample can generate microwaves resonantly with the ground-state spin, or, alternatively, a permanent magnet can be inserted to apply a static field along the optical axis. Appropriate mirrors and beam splitters allow for excitation through the objective by means of a continuous-wave 532-nm laser focused on the object plane. The fluorescence spectrum can be systematically measured as well as ODMR. The resonance peak shows a splitting ascribed to local strain distribution, and the frequency for RMW excitation is chosen at the point of maximum ODMR effect (see the Supplemental Material [49]).

Representative normalized $\mathrm{NV}^{-}$emission spectra in the SC and in FNDs deposited on a glass microscope coverslip are presented in Figs. 2(a) and 2(b), respectively, within each case the effect of perturbation by either RMW (left panes) or SMF (right panes) comparing low and high levels of optical excitation. The spectrum is dominated by the $\mathrm{NV}^{-}$emission from the zero-phonon line (ZPL) at $637 \mathrm{~nm}$ with phonon sidebands. The PL intensity is monotonously increasing with excitation intensity over the range of measurement, close to linear in most of the range but approaching saturation at the highest intensity values. Limited line-shape changes are noticeable from low to high power which may result from photoionization of $\mathrm{NV}$ defects or, alternatively, from saturation and/or thermal effects. At any of the excitation intensities, the $\mathrm{NV}^{0}$ spectral region remains unaltered by either RMW or SMF perturbation, while in the $\mathrm{NV}^{-}$emission, significant reductions are found resulting from the well-known spin dependence of the fluorescence efficiency. Here we set the applied microwave power close to maximum $(33 \mathrm{dBm})$ but avoiding saturation of the amplifier. The static field magnitude (approximately $220 \mathrm{mT}$ ) is chosen well above the critical value for saturation of the SMF contrast (see the Supplemental Material [49]). Throughout this work, we determine the induced contrast as the relative decrease in integrated intensity in the $\mathrm{NV}^{-}$emission range, 640-800 nm.

In Fig. 3, the experimental RMW and SMF contrasts are shown as a function of laser excitation intensity for 


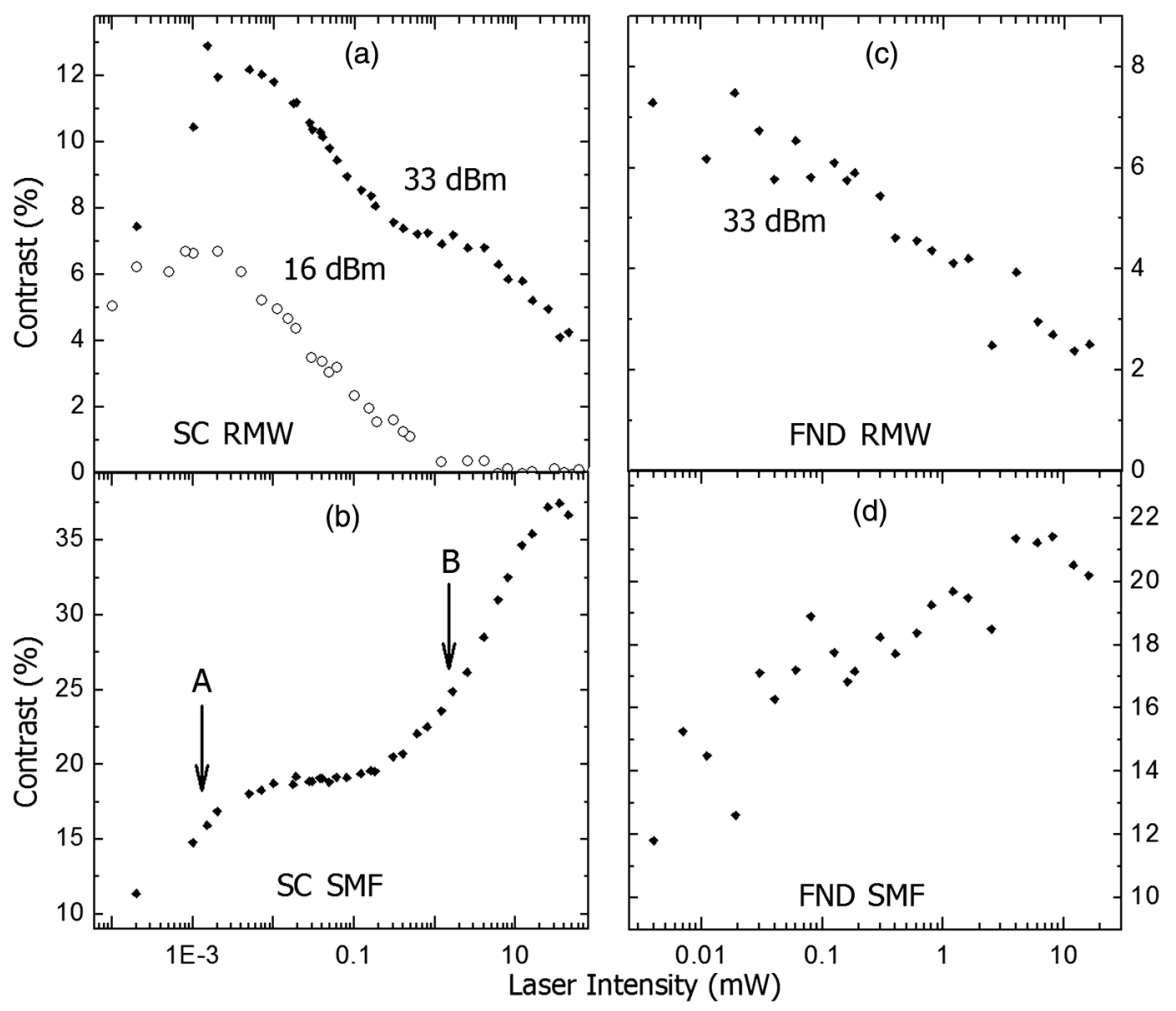

FIG. 3. Measured contrast as a function of optical excitation intensity for the FNDs (a),(b) and in SC (c),(d) perturbed by RMW (a), (c) and by SMF (b), (d). The RMW contrast in SC (c) is presented for a high and low level of applied microwave power. The $A$ and $B$ regions of transition discussed in the text are indicated in (b). either of the material systems. The measured contrasts are systematically lower in the FNDs than in the SC. While differences in the $\mathrm{NV}^{-}$dynamical properties in the two host materials may play a role, the random orientation of the defects in the FND case is an important factor, as we discuss in the next subsection. Also, a significant decrease is observed in RMW-induced contrast towards high optical excitation, in contrast with the marked and continuous increase seen in SMF-induced contrast. These opposite trends are found at high optical excitation both in the FNDs and the SC.

Looking more carefully, additional features come forward in the intensity dependence most evident in the SC case, which we, therefore, consider first. In the SC, the contrast is also measured at a microwave power of $16 \mathrm{dBm}$ (decreased by $17 \mathrm{~dB}$, i.e., $50 \times$ in power compared with $33 \mathrm{dBm}$ ). Clearly, the RMW contrast [Fig. 3(a)] is not decreasing monotonously, but an optimum excitation level appears, yielding 13\% (6.5\%) contrast at $33 \mathrm{dBm}$ $(16 \mathrm{dBm})$, occurring at a somewhat lower laser excitation level when lower RMW power is applied. The contrast is rapidly reducing towards lower laser intensities. At the high-power end, a decrease by a factor 3 is found under 33-dBm RMW, while under $16 \mathrm{dBm}$ it drops even quicker and vanishes around 1-mW laser power.

Considering the monotonously increasing SMF contrast [see Fig. 3(b)], one observes (i) an initial rise (marked $A$ ) towards a plateau value of $19 \%$ occurring close to the optimum in RMW contrast and (ii) another region of increase (marked $B$ ) to high values of up to $38 \%$ at the high-intensity end with an indication of saturation at the highest intensities. In the 33- $\mathrm{dBm}$ dependence for the SC, one may notice in the region around $1 \mathrm{~mW}$ a shoulder in the decreasing RMW contrast in the transition region $B$. From our analysis below, we show that the features $A$ and $B$ occur when the optical excitation rate becomes larger than the rate of spontaneous spin relaxation $1 / T_{1}$ and larger than the total decay rate of the intermediate singlet state $\left(k_{s}=k_{51}+2 k_{52}\right)$, respectively. In the literature, the $1 / T_{1}=160-350 \mathrm{~s}^{-1}$ value [44] and the $k_{s}=$ $1.7 \times 10^{6} \mathrm{~s}^{-1}$ value [43] are reported for diamond at room temperature. The measurements of the recovery of the PL intensity after abrupt interruption of RMW excitation in the limit of weak optical excitation point to similar $1 / T_{1}$ values in our materials (see the data in the Supplemental Material [49]). Considering the quite high $\mathrm{NV}$ and $\mathrm{N}$ densities in the studied layer, a $T_{2}$ of the order of $1 \mu \mathrm{s}$ can reasonably be expected [54]. The contrast curves for FNDs [Figs. 3(c) and 3(d)] are measured in a narrower range of optical intensities, and the data carry a larger experimental uncertainty due to the lower collected PL intensity compared with the SC. However, the same overall behavior is observed albeit with lower figures for contrast and with much less pronounced features. While the low-intensity limit cannot be as well covered, one notices an optimal RMW contrast of $7 \%$ followed by a strong reduction to $2 \%$ at high optical excitation. Conversely, the SMF contrast is increasing from $12 \%$ up to $21 \%$ for intense excitation, but here no clear stepping features can be discerned as are observed in the SC. As further discussed below, there is an 
essential difference between the SC and the FNDs. In the latter case, an ensemble of defects is excited, and one has to sum contributions over a quasirandom distribution of defect orientations.

\section{B. Rate-equations model for ground-state spin polarization}

We first consider the simplified energy-level scheme of the $\mathrm{NV}^{-}$defect [Fig. 1(a)] in zero magnetic field. Taking apart the microwave-driven spin nutation in the ground state, all other processes are treated as stochastic events, which can be described by rate equations for the populations in states of the five defect levels. However, the decay and relaxation rates listed in Table I vary over many orders of magnitude. In particular, the excited ${ }^{3} E$ states possess lifetimes that are 2 orders of magnitude shorter than the intermediate singlet level, and, thus, their decay can be considered as quasi-instantaneous with steadystate populations that are negligible under excitation applied in our experiments. Therefore, our model includes only the branching ratios expressing the importance of the ISC to the singlet levels relative to the direct decay to the ground state: $b_{1}=\left(k_{35} / k_{35}+k_{\mathrm{dir}}\right) \cong 0.10$ and $b_{2}=\left(k_{45} / k_{45}+k_{\text {dir }}\right) \cong 0.45$.

As shown in Appendix A, this treatment leads to a pair of linear differential equations for the distinct population $N_{1}\left(m_{S}=0\right)$ and $N_{2}$ (equal for $m_{S}=-1$ and +1 ) of the ${ }^{3} A_{2}$ levels by elimination of the population $N_{5}$ from the equations (using the condition $N_{5}=N_{\text {tot }}-N_{1}-2 N_{2}$, with $N_{\text {tot }}$ the number of $\mathrm{NV}^{-}$defects). Under steady-state conditions, one then derives a set of linear equations that can be readily solved. Figure 4 shows the steady-state populations as a function of optical excitation rate $W$ in the absence of RMW excitation (solid lines). Optical pumping produces spin polarization towards level $1\left(m_{S}=0\right)$, boosting the fluorescence efficiency. The balance with spontaneous spin relaxation $1 / T_{1}$ determines the initial increase of steady-state spin polarization, which reaches a plateau value determined by the selectivity of the ISC processes from the ${ }^{3} E$ to the intermediate singlet level as well as from the latter level to the ground ${ }^{3} A_{2}$ states. At high $W$, comparable to or larger than the decay rate $k_{5}=k_{51}+$ $2 k_{52}$ of the intermediate singlet, the ground levels are depleted, retaining, however, the spin polarization of the ground state, which increases even further.

\section{Contrast induced by resonant microwaves in the $\mathrm{SC}$}

For the RMW contrast, we consider the coherent oscillation of spin polarization $\left(N_{1} \leftrightarrow N_{2}\right)$ driven by the applied microwaves at or very close to resonance, combined with spin dephasing treated as a stochastic process (see Appendix B). This combination induces net average flows of population between the spin states which can be

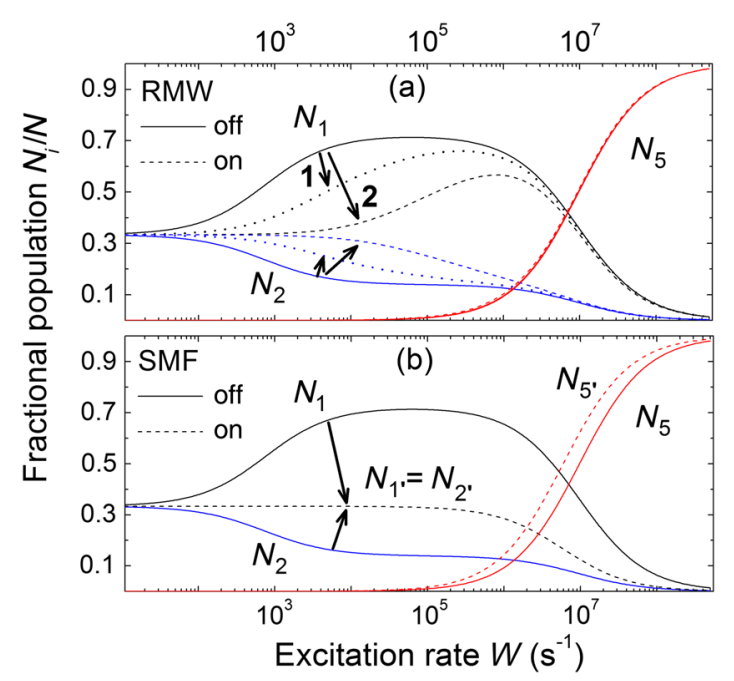

FIG. 4. Model calculations of the fractional populations as a function of optical excitation rate. The change in populations is marked by arrows in (a) under RMW with $\Omega_{1}=1.23 \times 10^{6} \mathrm{rad} / \mathrm{s}$ (marked 1) and $\Omega_{2}=7.6 \times 10^{6} \mathrm{rad} / \mathrm{s}$ (marked 2) and in (b) under SMF applied at the magic angle from the $\mathrm{NV}^{-}$defect axis. Results without (off) and with (on) applied perturbation are in solid and dotted lines, respectively.

included in the equations as transition rates depending on the Rabi frequency $\Omega$ and the total dephasing rate, $1 / T_{m}$. The latter involves intrinsic spin dephasing $1 / T_{2}$ as well as dephasing induced by optical excitation (proportional to $W b_{i}$, this involves the branching ratio $b_{i}$ accounting for loss of phase memory in ISC events only, not in direct decay to the ground state). The effective RMW-induced rates increase quadratically when $\Omega$ is small compared with the dephasing rate, as in our experiments. As a function of the optical excitation rate $(W)$, it is constant until $W b_{i}$ becomes comparable to the overall dephasing rate, above which it drops to zero. Switching on RMW decreases the spin polarization [see Fig. 4(a), broken lines] and, hence, partly restores the fluorescence. When at higher optical excitation, the overall dephasing rate $1 / T_{m}$ overthrows the Rabi frequency, and the RMW perturbation of the ground-state spin polarization becomes ineffective.

The fluorescence intensity is given by

$$
I_{\mathrm{fl}} \propto\left(1-b_{1}\right) N_{1}+2\left(1-b_{2}\right) N_{2}
$$

denoted here by $I_{\mathrm{fl}}^{\mathrm{off}}$ and $I_{\mathrm{fl}}^{\mathrm{on}}$ when calculated for zero and nonzero $\Omega$, respectively, which allows the RMW-induced contrast $C=\left(I_{\mathrm{fl}}^{\text {off }}-I_{\mathrm{fl}}^{\text {on }}\right) / I_{\mathrm{fl}}^{\text {off }}$ to be evaluated. The corresponding RMW-induced contrast curves [Fig. 5(a), broken lines] start from zero and rise steadily in the low excitation range, reflecting the increase in unperturbed polarization. This leads to a contrast that is increasing until the optically induced dephasing is taking over under more intense excitation and one finds a decrease in contrast, as shown 

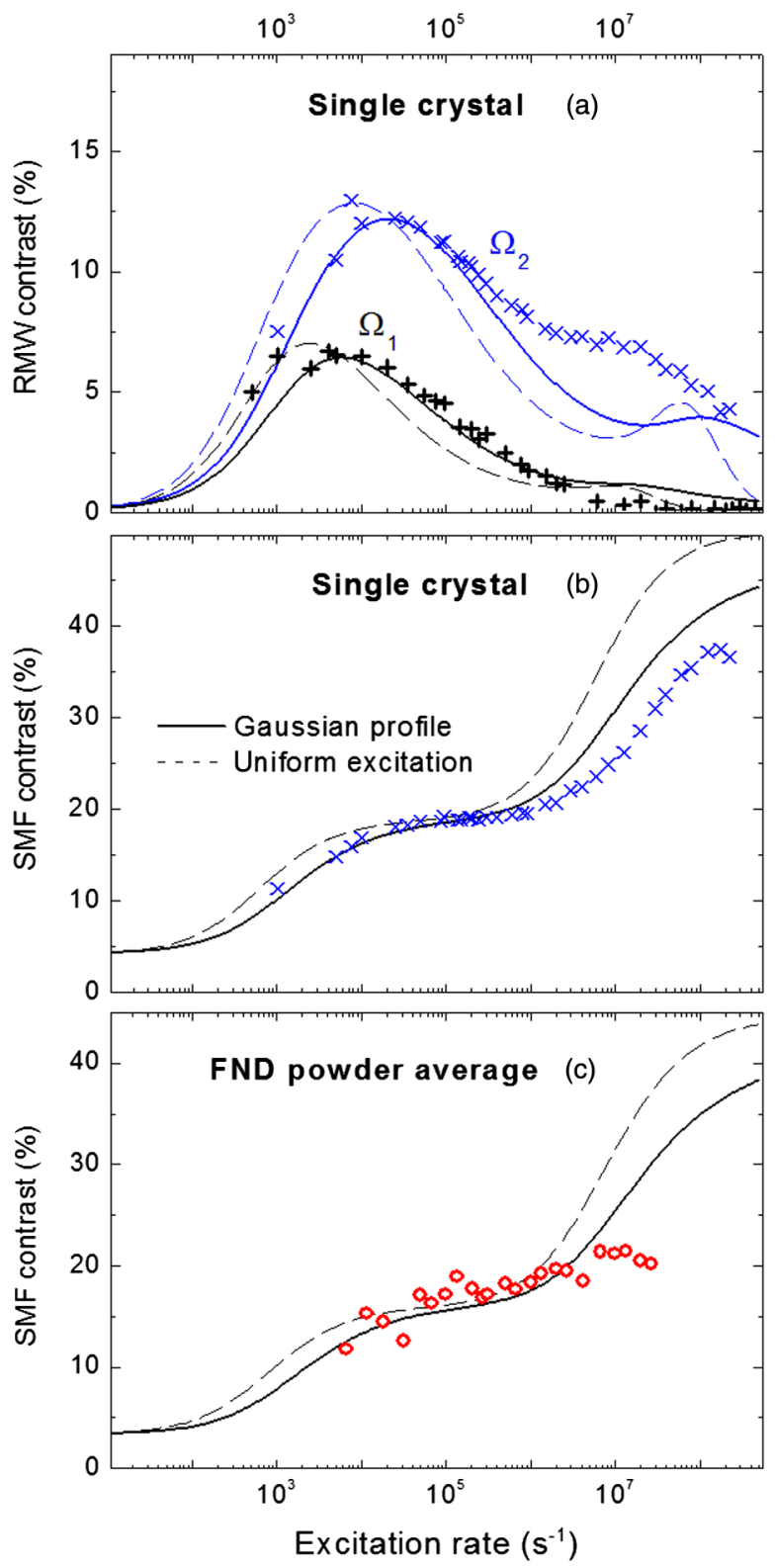

FIG. 5. Model calculations of induced contrast as a function of optical excitation rate in SC (a) under RMW with Rabi frequencies $\Omega_{1}=1.23 \times 10^{6} \mathrm{rad} / \mathrm{s}, \Omega_{2}=7.6 \times 10^{6} \mathrm{rad} / \mathrm{s}$ corresponding to 16 and $33 \mathrm{dBm}$, respectively, and (b) under SMF applied at the magic angle from the $\mathrm{NV}^{-}$defect axis as in the $\mathrm{SC}$ experiments. (c) Contrast in the FND sample assuming averaging over the defect orientation with strong light scattering in the ND aggregates. The results for uniform excitation intensity (dashed line) are compared to those for a Gaussian intensity profile (solid line; see text). After linear scaling of the abscissa (factor: $5 \times 10^{6} \mathrm{~s}^{-1} / \mathrm{mW}$ for $\mathrm{SC}$ and $1.67 \times 10^{6} \mathrm{~s}^{-1} / \mathrm{mW}$ for FNDs), the measured data points are inserted for comparison.

in Fig. 5(a). The Rabi frequencies are experimentally determined as described in the Supplemental Material [49] to be $\Omega_{1}=1.23 \times 10^{6} \mathrm{rad} / \mathrm{s}$ and $\Omega_{2}=7.6 \times 10^{6} \mathrm{rad} / \mathrm{s}$, which correspond to microwave magnetic fields $B_{1}$ on the sample of 0.031 and $0.189 \mathrm{mT}$ for the 16- and $33-\mathrm{dBm}$ measurements, respectively. The ratio $\left(\Omega_{2} / \Omega_{1}\right)$ falls slightly short of the value $\sqrt{50}$ expected for the nominal $17-\mathrm{dB}$ difference in microwave powers, but this minor deviation is pointing only to weak saturation of the microwave amplifier. There is good agreement with the experimental contrast data: the maximum contrast values are matched, and the initial rise as well as the position and width of the maximum are well reproduced. Beyond this maximum, the experimental data under $33 \mathrm{dBm}$ display a shoulder in the curve around $W=10^{7} \mathrm{~s}^{-1}$, and this does not perfectly correspond with the calculation. Nevertheless, in both simulations (for 16 and $33 \mathrm{dBm}$ ), there is a contribution in the region labeled $B$ in Fig. 3(b). This is where the SMF contrast shows a second rise at the same time, which is correlated with the buildup of population in the intermediate singlet state $N_{5}$ (see Fig. 4).

It is worth mentioning here that according to the model calculation, the only effect of further increase of microwave power is broadening of the maximum as a function of excitation intensity and contrast does not become significantly higher. Similarly, no gain in maximum contrast is found upon decreasing the width of the inhomogeneous distribution in the calculation.

At this point, it should be remembered that the measurements are performed under focused-laser-beam conditions which leads to a Gaussian transversal intensity distribution of the light in the focus. This implies that at a certain nominal peak intensity in the center of the spot, $\mathrm{NV}^{-}$defects in its periphery are subject to weaker excitation. Integration of the previous results over this distribution for a given peak excitation rate $W$ is quite straightforward and yields the solid curves in Fig. 5(a). Indeed, the tails of the contrast curves at higher intensities are now more extended, coming closer to the experimentally observed behavior.

\section{Contrast induced by a static magnetic field in the SC}

SMF-induced contrast results from the different spin eigenstates, which in zero field are quantized along the trigonal defect axis, while a different quantization is imposed by a sufficiently strong static magnetic field (close to full quantization for approximately $220 \mathrm{mT}$ at magic angle: pure to $>96 \%$ and $>98 \%$ in the ground and excited triplet, respectively). The modified energylevel diagram is schematically shown in Fig. 1(b). The field-quantized spin states $\left|m_{S}^{\prime}\right\rangle$ can be expressed in terms of the zero-field states $\mid m_{S}>$ by mixing coefficients depending on the angle $\theta$ between the SMF and the defect axis. In a simplified treatment (e.g., the Appendix of Ref. [47]), the decay rates under SMF can then be derived from those in zero field (see Fig. 1 and Table I) by the expressions 


$$
\begin{aligned}
& k_{35}^{\prime}=\left(1-\sin ^{2} \theta\right) k_{35}+\sin ^{2} \theta k_{45}, \\
& k_{45}^{\prime}=\frac{1}{2} \sin ^{2} \theta k_{35}+\left(1-\frac{1}{2} \sin ^{2} \theta\right) k_{45}, \\
& k_{51}^{\prime}=\sin ^{2} \theta k_{35}+\left(1-\sin ^{2} \theta\right) k_{52}, \\
& k_{52}^{\prime}=\left(1-\frac{1}{2} \sin ^{2} \theta\right) k_{51}+\frac{1}{2} \sin ^{2} \theta k_{52} .
\end{aligned}
$$

Considering that in ground as well as in the excited triplet the $m_{S}^{\prime}=-1$ and +1 states are treated symmetrically $\left(N_{2^{\prime}}=N_{2^{\prime} \pm}\right)$, the rate equations for the populations $N_{1^{\prime}}$ and $N_{2^{\prime}}$ become identical to those of the zero-field situation, adopting now the modified decay rates in Eq. (2) and, accordingly, change branching ratios for evaluation of the fluorescence intensity in Eq. (1). Note that the expressions are independent of the magnitude of the magnetic field in the high-field approximation, which is expressed in the full quantization of the spin states along this field. The emission intensities are calculated using the steady-state solutions for the field-modified equations and then compared with the zero-field results in order to evaluate the SMF-induced contrast, similar to the treatment for RMWs.

In Fig. 5(b), we show (solid line, black) the intensity dependence of the SMF contrast for the case $\theta=54.74^{\circ}$, corresponding to the experimental SC case where the applied field is along a $\langle 100\rangle$ crystal axis at the same angle from any of the $\langle 111\rangle$ defect axes. Note that for this special case, the optically induced spin polarization vanishes $\left[N_{1^{\prime}}=N_{2^{\prime}}\right.$; see, also, in Fig. 4(b)]. Obviously, the main features observed in the experiment [Fig. 3(b)] are recovered in this calculation using parameters from the literature (Table I), with some of them varying only within their range of experimental uncertainty. Only one parameter is freely adjustable, i.e., the proportionality factor between experimental laser power and excitation model parameter $W$. The value $4 \times 10^{6} \mathrm{~s}^{-1} / \mathrm{mW}$ yields the best agreement for the positions of low- and high-intensity steps in the SMF-contrast curve. Correlation of the transition regions in $W$ with the rates of physical processes allows us to discern the origin of these two steps in the curves: competition of the optical excitation $(W)$ with spin relaxation in the ground state $\left(1 / T_{1}\right)$ and with decay rate from the intermediate singlet $\left(k_{s}=k_{51}+2 k_{52}\right)$. The latter effect is also obvious from the marked increase in population $N_{5}$ of this level, as shown in Fig. 4.

The presented rate-equations model for $\mathrm{NV}^{-}$photodynamics quite successfully explains the intensity dependence of the RMW and SMF contrasts in SC experiments. The respective opposite trends at high intensity are obtained as a straightforward result. Quantitative agreement is obtained for the SMF contrast at all excitation levels including the step features. Also, for the RMW contrast, very good quantitative agreement is reached, with only a small discrepancy at higher excitation rates: the calculation yields a bump that is narrower and appears at higher rates than observed in the contrast curves.

\section{E. Effects of orientational averaging in FNDs}

Considering that for the FND experiments a large number of nanocrystals, each providing four possible orientations of the $\mathrm{NV}^{-}$defect in the lattice, are deposited on the glass substrate from the solution, it is reasonable to assume a quasirandom orientation of the defect symmetry axis in these measurements. The orientation has to be averaged with respect to the polarization vector of the light (only the component perpendicular to the defect axis is active) and to either the static magnetic field (orientationdependent mixing) or the oscillating magnetic field of the RMWs (transitions only from the perpendicular component). It should be noted that the SC experiments, as we perform them, escape such complications since all of the corresponding vectors are aligned in $\langle 100\rangle$ crystal directions either perpendicular (for the static and microwave magnetic fields) or in plane with respect to the crystal slab. In these configurations, the four defect orientations are equivalent. It is also worth mentioning that the measurements in the FND sample are not performed over the whole intensity range because of the lower overall signal, setting a useful low-power limit 20 times above that for the SC.

It is now straightforward to recalculate the dependence of the SMF at different angles between the static field and defect axis using the transformation of the rate parameters given in Eq. (2). This recalculation results in essentially the same features as for the earlier magic angle calculation for the SC experiments, only with decreased contrast values for smaller angles (vanishing towards the parallel configuration) as well as for the larger angles towards the perpendicular case. In any case, the two steps in the increasing contrast with the optical transition rate occur around the same characteristic $W$ values which are rationalized to be connected with the spin population relaxation time $T_{1}$ and with the lifetime of the intermediate singlet state $1 / k_{s}$. However, it should be realized that a given laser intensity will lead to different excitation rates for FNDs in different orientations. Therefore, the abscissa in these graphs has to be rescaled accordingly for each orientation. The scaling factors range from zero to a maximum value when the polarization and defect axis are varied from parallel to perpendicular, respectively. Also, light scattering in the nanocrystals is scrambling the light polarization. A calculation is performed using the same parameters for the $\mathrm{NV}^{-}$properties (see Table I), averaging over independent random orientations of both defect axis and polarization of the light. The latter corresponds to strong light scattering in the ND aggregates. (An alternative calculation that assumes random defect orientation combined with the unaltered polarization of the laser light gives very similar results.) Quite remarkably, the reduction in SMF contrast is not very important from the single-crystal case to the one with 
random orientations. Only a limited angular region around parallel orientation has low SMF contrast, and this region turns out to have a low contribution in the orientational average. A very good agreement is found when the experimental data points are inserted in the graph assuming a local excitation intensity reduced by a factor 3 relative to the SC case, as is expected from strong light scattering.

Random orientation of the microwave field $\left(B_{1}\right)$ results in different Rabi frequency for different orientations of the defects and, hence, a superposition of contrast curves for a wide variation of this parameter from zero to full effectivity $\left(\Omega \propto B_{1} \cos \theta\right)$. This superposition combined with the previously discussed intricacies concerning polarization form together a complex combined problem, and we do not attempt modeling. Obviously, the treatment for the case of single-particle or single-center experiments will be greatly simplified. Our experiment in FNDs, in spite of limited SNR, shows similar behavior as that of the SC but with a broader and lower maximum in the contrast [see Fig. 3(c)].

\section{F. SMF contrast applied in FND fluorescence microscopy of neuronal cell cultures}

The contrast induced by a static magnetic field is demonstrated here to be higher than by resonant microwaves; moreover, it is increasing monotonously with optical excitation power. The approach is straightforward and robust for application in various microscope modalities and provides a tool for the exploration of targeted introduction of functionalized nanodiamond probes in various cell cultures. Very recently, different groups $[7,55,56]$ have investigated the effects of in vitro and in vivo treatment of neurons with nanodiamonds and discussed their potential $[4,57]$ among other nanoparticle-based probes and sensors. In this line of research, here we demonstrate the SMFcontrast method in several microscope configurations for a nontrivial system of high interest consisting of neuron cell cultures incubated with FNDs.

First, we combine the SMF-contrast imaging with imaging of living neuronal culture (see Fig. 6), which is performed in a setup suitable for parallel patch-clamp experiments. After acquiring the image of the neurons under infrared DIC [Fig. 6(a)], fluorescence imaging is performed with 532-nm laser illumination through the objective and appropriate red filtering in the collection light path [Fig. 6(b)]. Subtraction of fluorescence images with and without SMF [permanent magnet inserted and removed; see Fig. 1(c)] yields strong selectivity for the FNDs [Fig. 6(c); also in the Supplemental Material [49]; slices through images in Figs. 6(b) and 6(c) illustrating background elimination]. We find it useful to sandwich recordings with applied SMF between two takes without the field to ascertain reproducibility and to eliminate eventual bleaching effects in the background by averaging between the first and last image. We overlay the image from SMF contrast on the DIC image to show the colocalization of the nanodiamonds and neuronal cell structural elements [see Fig. 6(d)]. In a defocusing series in both DIC and SMF contrast (in the Supplemental Material [49]), we establish a correlation between the sharpness of the images, which is pointing to colocalization of the FNDs with the cell sidewalls.

In a second example, background emission is intentionally introduced by labeling neurons with a fluorescent reporter protein enabling discrimination of neurons from glial cells upon fixation of the samples. The dye emission in the green has a significant tail into the FND spectral region and residual weak excitation is found even at $532 \mathrm{~nm}$. In an inverted microscope setup, we take images under green LED illumination (see Fig. 7) in a SMF-contrast sequence as we discuss above. The detected intensity without field shows the stained neural structures, and the difference image reveals the presence of well-defined spots with FND
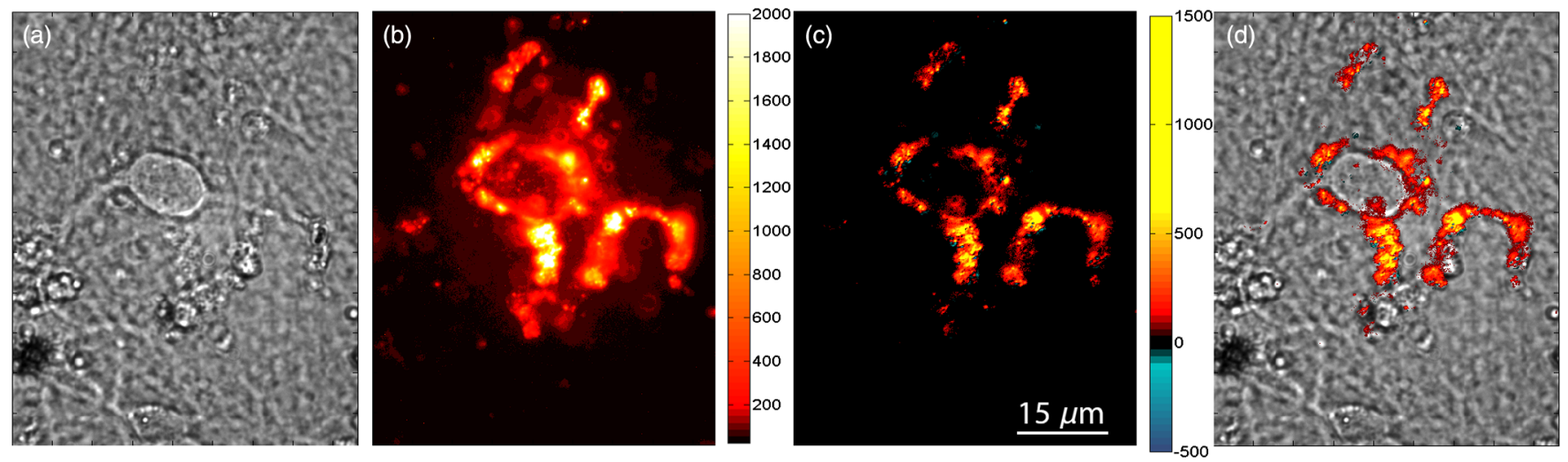

FIG. 6. Imaging of a FND-seeded neuronal culture (a) in DIC contrast, (b) in intensity of fluorescence intensity in the $\mathrm{NV}^{-}$spectral $^{-1}$ region (defocused, 2-mW, 532-nm laser illumination), and (c) in SMF contrast of FND fluorescence discriminating FND emission from spurious background in the laser-illuminated area. In (d), the DIC (gray) and SMF contrast (false color) images are overlaid. Color code shown for (b) and (c). (See, also, the Supplemental Material [49].) 

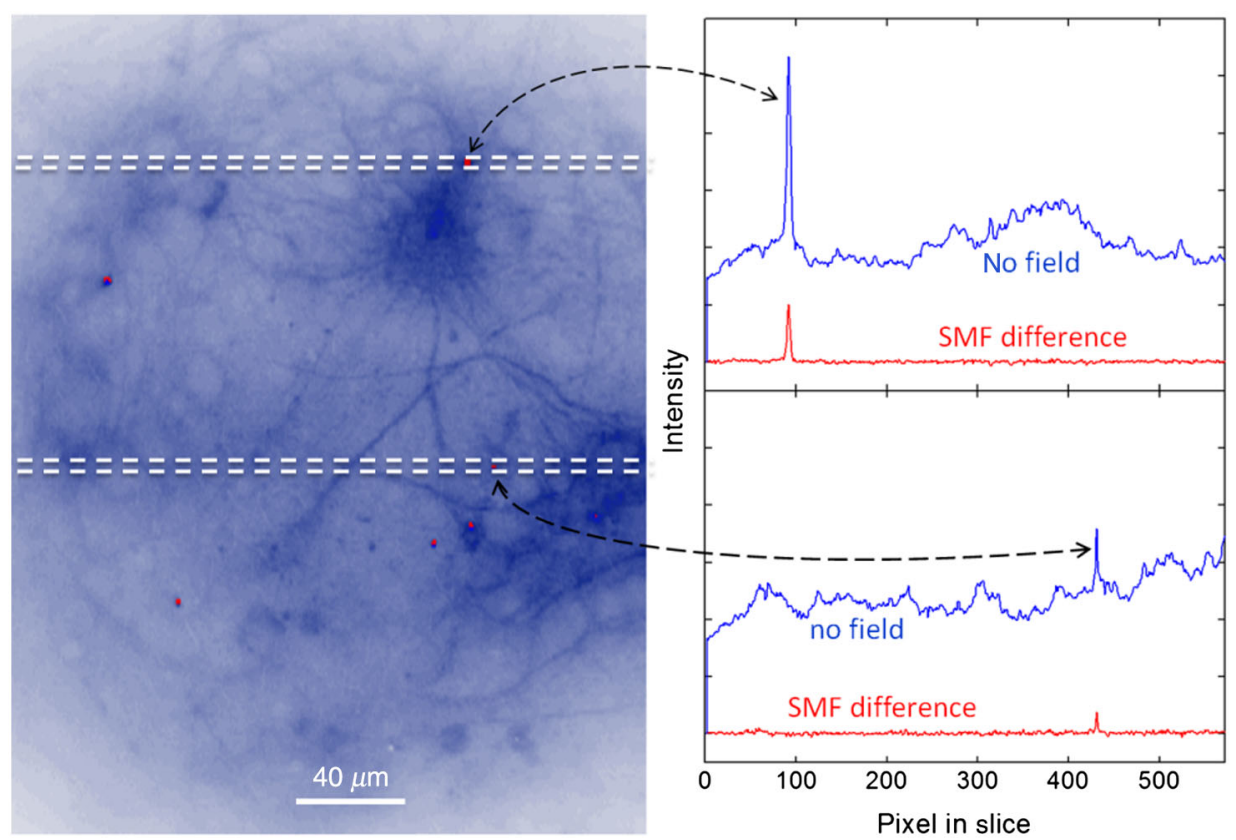

FIG. 7. Left: The image of a FND-seeded neuronal culture, which was immunolabeled and subsequently fixated. The image was taken in the inverted microscope with $40 \times$ objective under filtered 525-nm LED illumination (1 $\mathrm{mW}$ on sample). The red spots show the presence of FNDs as derived from the difference image taking advantage of SMF contrast. Right: For two selected spots, the signal without field and the SMF difference signal are plotted for the indicated horizontal slices which shows the efficient discrimination of FND fluorescence from the staining background.
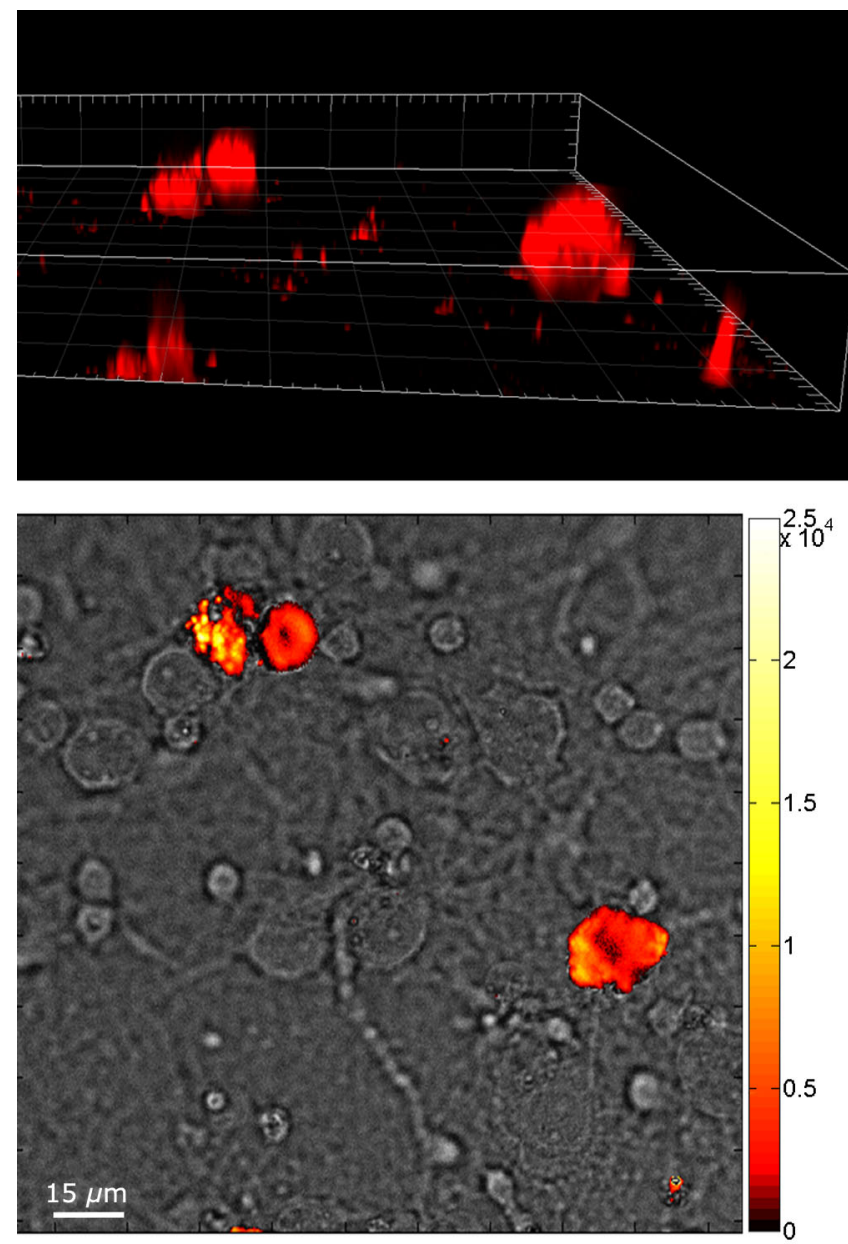

FIG. 8. Top: Perspective view of FND agglomerates in a neuronal culture measured by confocal fluorescence microscopy in vertically $1-\mu \mathrm{m}$ spaced slices. Bottom: Overlay of an image of the seeded cell culture in FND fluorescence (with color code bar) with the corresponding one in bright-field contrast. fluorescence showing the SMF contrast. The effectiveness of the approach is illustrated in the graphs on the right of Fig. 7, which show selected profiles through the image before and after subtraction with contrast reaching over $30 \%$ in the FND spots. In these measurements, a sufficiently intense local optical excitation is required to obtain appropriate SMF-contrast values, which is illustrated in a set of measurements performed in the upright biological microscope on a similar stained sample with small FND aggregates (see the Supplemental Material [49]). Indeed, for the same incident laser intensity, a much higher contrast is reached using the $63 \times$ objective providing approximately $40 \times$ more concentrated optical power than obtained with the $10 \times$ objective.

Finally, confocal imaging of FNDs in a living neuronal culture is demonstrated in Fig. 8(a) (top), which shows a perspective view of the accumulation of nanoparticles in specific regions. The regions of FND accumulation display SMF-contrast values in the 10\%-15\% range, possibly limited by the available level of green light excitation from the standard illumination accessory. We detect FNDs within the measured $20-\mu \mathrm{m}$ section $(1-\mu \mathrm{m}$ steps $)$, mainly in the lower part closer to the glass substrate. Colocalized with cellular structures is observable in the overlay with the bright-field image of the culture [Fig. 8(b); see, also, in the Supplemental Material [49] a series of frames from the $z$ scan and additional 3D views).

\section{CONCLUSIONS}

The comparison of contrast induced either by RMWs or by a SMF in the fluorescence of $\mathrm{NV}^{-}$centers in diamond reveals significant differences between both approaches under increasing optical excitation intensity. The SMF 
approach yields a high contrast that is continuously increasing (over 35\% in the SC and 20\% in FNDs), while the RMW approach offers a more limited maximum contrast, reaching this at quite moderate optical excitation levels. The SMF approach, therefore, offers clear advantages for background-free nanodiamond detection in a biological environment by which the selective introduction can be monitored of functionalized FNDs in primary cell cultures (e.g., specifically targeting carcinoma). For living cells, the SMF-contrast method also avoids thermal perturbation by the deposition of microwave energy in the aqueous intra- and extracellular medium. The incorporation of the method in standard biological microscopes is straightforward as we show here for observation of living and fixated neuronal cultures in three different instrumental combinations, including the opportunity of parallel electrophysiology measurements in the patch-clamp setup. In a more general framework, the SMF detection can also be applied as an initial monitoring method combined with sophisticated sensor functions (electrical and magnetic, thermometry, local ion potential) already demonstrated and envisaged for the FNDs. The respective ODMR-based protocols can then be applied in an (eventually prolonged) off period of the SMF-contrast sequence.

To avoid the insertion of the permanent magnet and eliminate the possible disturbing vibrations, a compact electromagnet can be applied. In previous work by Sarkar et al. [40], this was demonstrated with applied fields of approximately $10 \mathrm{mT}$, which should, however, be increased to $>50 \mathrm{mT}$ in order to reach optimal contrast. The improvement in SNR using such an on-off modulation treatment of the images [40] can be fruitfully combined with the optimal selection based on our analysis of applied field and excitation intensities. Also, with the high brightness of the $\mathrm{NV}^{-}$emission, it will often prove advantageous to reduce the duty cycle of optical excitation in order to combine selectivity derived from high values of the contrast with acceptable average power onto the sample. The required power management can be easily implemented by modulation of either laser or LED sources. Similarly, in confocal microscopy, one can judiciously combine optical excitation intensity with collection time per pixel to optimize SMF contrast for background-free biological imaging.

\section{ACKNOWLEDGMENTS}

The authors are grateful to D. Van Dyck, M. Wijnants, and P. Casteels for technical assistance, and B. Yavkin for contributions to the implementation of ODMR measurements. Financial support for this research is kindly acknowledged from the Research Foundation-Flanders (FWO, Grant No. G.088812N), the European Commission (FP7-PEOPLE-IEF INCA-NANEP, Contract No. 328214, and the FP7/2007-2013 project DIADEMS, Grant Agreement No. 611143), the Belgian Science Policy Office (BELSPO), the Ministry of Science and
Education of the Czech Republic (Contract No. CZ.1.07/ 2.3.00/20.0306).

\section{APPENDIX A: RATE-EQUATIONS MODEL FOR THE NV ${ }^{-}$CENTER UNDER OPTICAL EXCITATION}

Starting from the level scheme in Fig. 1(a), we define the populations for the ground ${ }^{3} A_{2}$ triplet as $N_{1}\left(m_{S}=0\right)$ and $N_{2}^{ \pm 1}\left(m_{S}= \pm 1\right)$ for the excited ${ }^{3} E$ triplet $N_{3}\left(m_{S}=0\right)$ and $N_{4}^{ \pm}\left(m_{S}= \pm 1\right)$, and finally $N_{5}$ for the lowest intermediate singlet level. However, in zero field and assuming unpolarized optical excitation, there can be no difference between the $m_{S}= \pm 1$ cases, and we can write $N_{2}=N_{2}^{ \pm}$ and $N_{4}=N_{4}^{ \pm}$. We assume that the optical excitation rate $W=W_{13}=W_{24}$ is the same for the allowed (i.e., $m_{S}$ conserving) transitions from ${ }^{3} A_{2}$ to ${ }^{3} E$, while forbidden transitions are not considered. Similarly, between the triplets, we include only decay rates for the allowed transitions with rate constant $k_{\mathrm{dir}}=k_{31}=k_{42}$ assumed equal. Taking into account the short lifetime of the excited triplet states, we ignore their populations and consider the excitation and subsequent relaxation to either ground triplet or intermediate singlet as quasi-instantaneous. The ISC rate constants $k_{35}$ and $k_{45}$ appear now in the branching ratios $b_{1}=k_{35} /\left(k_{35}+k_{\mathrm{dir}}\right)$ and $b_{2}=k_{45} /\left(k_{45}+k_{\mathrm{dir}}\right)$ giving the fraction of excited centers feeding the singlet population $N_{5}$. These assumptions lead us to the rate equations

$$
\begin{aligned}
\frac{d N_{1}}{d t} & =k_{\mathrm{sp}}\left(N_{2}^{+}+N_{2}^{-}-2 N_{1}\right)-W b_{1} N_{1}+k_{51} N_{5} \\
& =2 k_{\mathrm{sp}}\left(N_{2}-N_{1}\right)-W b_{1} N_{1}+k_{51} N_{5}, \\
\frac{d N_{2}}{d t} & =\frac{d N_{2}^{ \pm}}{d t}=k_{\mathrm{sp}}\left(N_{1}-N_{2}^{ \pm}\right)-W b_{2} N_{2}^{ \pm}+k_{52} N_{5} \\
& =k_{\mathrm{sp}}\left(N_{1}-N_{2}\right)-W b_{2} N_{2}+k_{52} N_{5}, \\
\frac{d N_{5}}{d t} & =W\left[b_{1} N_{1}+b_{2}\left(N_{2}^{+}+N_{2}^{-}\right)\right]-\left(k_{51}+2 k_{52}\right) N_{5} \\
& =W\left(b_{1} N_{1}+2 b_{2} N_{2}\right)-\left(k_{51}+2 k_{52}\right) N_{5},
\end{aligned}
$$

in which $k_{\mathrm{sp}}$ is the spontaneous relaxation rate between the $m_{S}=0$ and each of the $m_{S}= \pm 1$ ground states. In the case without optical excitation $\left(W=0, N_{5}=0\right)$, one can easily derive the relaxation of ground-state spin polarization $(d / d t)\left(N_{2}-N_{1}\right)=-3 k_{\mathrm{sp}}\left(N_{2}-N_{1}\right)$ relating the relaxation rate to the usual population relaxation rate $1 / T_{1}=3 k_{\mathrm{sp}}$.

In steady-state conditions and assuming constant total population $N_{\text {tot }}$ (substituting $N_{5}=N_{\text {tot }}-N_{1}-2 N_{2}$ ), the rate equations are reduced to a set of two inhomogeneous linear equations for the fractional populations of the states $n_{i}=N_{i} / N_{\text {tot }}$, 


$$
\begin{array}{r}
\left(2 k_{\mathrm{sp}}+W b_{1}+k_{51}\right) n_{1}+2\left(k_{51}-k_{\mathrm{sp}}\right) n_{2}=k_{51}, \\
\left(k_{52}-k_{\mathrm{sp}}\right) n_{1}+\left(k_{\mathrm{sp}}+W b_{2}+2 k_{52}\right) n_{2}=k_{52},
\end{array}
$$

with explicit solutions

$$
n_{1}=\left|\begin{array}{cc}
k_{51} & 2\left(k_{51}-k_{\mathrm{sp}}\right) \\
k_{52} & k_{\mathrm{sp}}+W b_{2}+2 k_{52}
\end{array}\right| / D
$$

and

$$
n_{2}=\left|\begin{array}{cc}
2 k_{\mathrm{sp}}+W b_{1}+k_{51} & k_{51} \\
k_{52}-k_{\mathrm{sp}} & k_{52}
\end{array}\right| / D
$$

with

$$
D=\left|\begin{array}{cc}
2 k_{\mathrm{sp}}+W b_{1}+k_{51} & 2\left(k_{51}-k_{\mathrm{sp}}\right) \\
k_{52}-k_{\mathrm{sp}} & k_{\mathrm{sp}}+W b_{2}+2 k_{52}
\end{array}\right| .
$$

For the contrast calculations, these equations and solutions remain essentially the same under either SMF or RMW, except for suitable changes in the parameters involved.

For a large static magnetic field (Zeeman interaction large compared to ZFS) at an angle $\theta$ from the trigonal $\mathrm{NV}^{-}$ defect axis, one assumes a probability of ISC to be a superposition of those from or to each of the zero-field eigenstates weighed with their contribution in the eigenstates quantized along the field. Taking into account that the Zeeman splitting is still small compared to thermal energy at room temperature and that light excitation dominates the spin-polarization effects, equal populations $N_{2^{\prime}}=N_{2^{\prime}}^{ \pm}$can still be assumed. The only difference between the calculations for RMW and SMF perturbation is the replacement of the rate constants $k_{i j}$ by the $k_{i j}^{\prime}$ given in Eq. (2). Steadystate populations $N_{1^{\prime}}$ and $N_{2^{\prime}}$ and, thus, the spin polarization, are now dependent on orientation. In the special case of single-crystal experiments with field along a $\langle 100\rangle$ crystal direction $\left(\theta=54.74^{\circ}\right.$ for the four possible defect orientations), the state mixing leads to a specific situation. The ISC parameters towards the singlet level become equal $k_{35}^{\prime}=k_{45}^{\prime}$ and also from the singlet to the ground states $k_{51}^{\prime}=k_{52}^{\prime}$, which leads to vanishing spin polarization in the ${ }^{3} A_{2}$ ground level, $N_{1^{\prime}}=N_{2^{\prime}}$ (see Fig. 4(b)). In other cases, summing over defect orientations is required, and in FND ensembles, one can expect a quasirandom orientational distribution.

For the RMW case, the only change in the calculations is the rate constant $k_{\mathrm{sp}}$ for population relaxation between levels 1 and 2 which is replaced by an effective relaxation rate $k_{\text {sp. }}$. The latter rate depends on the Rabi frequency at the applied RMW excitation level and on the rate of dephasing processes, as is derived in Appendix B.

\section{APPENDIX B: RMW-induced effective relaxation rates}

Under exactly resonant microwave excitation in the absence of external magnetic field, the spin in the triplet ground state is driven by the microwaves in a coherent oscillation at the Rabi frequency $\Omega$ between the states of levels 1 and 2 . $\mathrm{A} \mathrm{NV}^{-}$defect appearing after an excitation cycle in level $1\left(m_{S}=0\right)$ at time $t_{g}$ is found with probabilities $P_{1}^{(1)}=\cos ^{2} \Omega\left(t-t_{g}\right)$ and $P_{2}^{(1)}=\sin ^{2} \Omega\left(t-t_{g}\right)$ in the same level and in level $2\left(m_{S}= \pm 1\right)$, respectively, until the state is reset by a dephasing event. This microwave-driven oscillation will at time $t$ contribute flows of population $(d / d t)\left(P_{1}^{(1)}\right)=$ $-\Omega \sin 2 \Omega\left(t-t_{g}\right),(d / d t)\left(P_{2}^{(1)}\right)=+\Omega \sin 2 \Omega\left(t-t_{g}\right)$ from $1 \rightarrow 2$ and vice versa, respectively. The same considerations apply to defects starting in level 2, with population coherently oscillating between the two levels. The probability that this process effectively contributes is $1 / T_{m} e^{-\left(t-t_{g}\right) / T_{m}}$ for an exponential process with total dephasing rate $1 / T_{m}$. In steady-state conditions, there is a constant rate of appearance in the past, over which we can integrate transition probabilities between the levels, leading to an increased population relaxation rate due to the combined effect of RMW and dephasing:

$$
\begin{aligned}
k_{\mathrm{sp}}^{\prime} & =k_{\mathrm{sp}}+\left(\Omega / T_{m}\right) \int_{-\infty}^{t} d t_{g} \sin 2 \Omega\left(t-t_{g}\right) e^{-\left(t-t_{g}\right) / T_{m}} \\
& =k_{\mathrm{sp}}+\frac{2 \Omega / T_{m}}{(2 \Omega)^{2}+\left(1 / T_{m}\right)^{2}} \Omega .
\end{aligned}
$$

Considering near-resonant conditions, with transition frequency $\delta \omega$ away from the microwave frequency, the Rabi oscillation will happen at a modified frequency $\Omega^{\prime}=\sqrt{\Omega^{2}+\delta \omega^{2}}$ and over a more limited angle of $\Phi=\pi-2 \tan ^{-1}(1|\delta \omega / \Omega|)$ away from the poles of the Bloch sphere, proportionally reducing the spin population flows by a factor $(1-\cos \Phi) / 2$, leading to the final expression:

$$
k_{\mathrm{sp}}^{\prime}=k_{\mathrm{sp}}+\frac{1-\cos \Phi}{2} \frac{2 \Omega^{\prime} / T_{m}}{\left(2 \Omega^{\prime}\right)^{2}+\left(1 / T_{m}\right)^{2}} \Omega^{\prime} .
$$

The ODMR resonance line in zero field is dominated by an inhomogeneous distribution of the transition frequency from $\mathrm{NV}^{-}$centers with varying surroundings. Their contributions to the intensities without or with RMW or SMF are summed over a distribution of $\delta \omega$ values derived from the experimental ODMR line shape (see the Supplemental Material [49]). For an inhomogeneous width larger than the Rabi frequency, as is the case here, the effect of detuning within the linewidth is important. All processes responsible for dephasing can be included in $1 / T_{m}$ : the pure spin dephasing $1 / T_{2}$ but also the result of optical excitation. If any optical excitation is considered to induce dephasing, 
$1 / T_{m}=1 / T_{2}+W$. Evidently, ISC leads to loss of phase memory. However, phase memory can be expected throughout the processes of excitation and prompt return to the ground state. In that case, the dephasing effect of optical excitation must be reduced by the branching ratio $1 / T_{m, i}=1 / T_{2}+W b_{i}(i=1,2)$, resulting in different values for $k_{\mathrm{sp}, i}^{\prime}$ for the population relaxation processes starting from each of the two levels. In our calculations, the latter assumption is applied. However, only quite small differences are found when we compare with calculations including full dephasing under optical excitation.

[1] C.-C. Fu, H.-Y. Lee, Kowa Chen, T.-S. Lim, H.-Y. Wu, P.-K. Lin, P.-K. Wei, P.-H. Tsao, H.-C. Chang, and W. Fann, Characterization and application of single fluorescent nanodiamonds as cellular biomarkers, Proc. Natl. Acad. Sci. U.S.A. 104, 727 (2007).

[2] E. Perevedentseva, Y.-C. Lin, M. Jani, and C.-L. Cheng, Biomedical applications of nanodiamonds in imaging and therapy, Nanomedicine 8, 2041 (2013).

[3] R. Schirhagl, K. Chang, M. Loretz, and C. L. Degen, Nitrogen-vacancy centers in diamond: Nanoscale sensors for physics and biology, Annu. Rev. Phys. Chem. 65, 83 (2014).

[4] A. M. Monaco and M. Giugliano, Carbon-based smart nanomaterials in biomedicine and neuroengineering, Beilstein J. Nanotechnol. 5, 1849 (2014).

[5] W. W.-W. Hsiao, Y. Y. Hui, P.-C. Tsai, and H.-C. Chang, Fluorescent nanodiamond: A versatile tool for long-term cell tracking, super-resolution imaging, and nanoscale temperature sensing, Acc. Chem. Res. 49, 400 (2016).

[6] Y. Kuo, T.-Y. Hsu, Y.-C. Wu, and H.-C. Chang, Fluorescent nanodiamond as a probe for the intercellular transport of proteins in vivo, Biomaterials 34, 8352 (2013).

[7] T.-C. Hsu, K.-K. Liu, H.-C. Chang, E. Hwang, and J.-I. Chao, Labeling of neuronal differentiation and neuron cells with biocompatible fluorescent nanodiamonds, Sci. Rep. 4, 5004 (2014).

[8] I. Rehor, J. Slegerova, J. Kucka, V. Proks, V. Petrakova, M.-P. Adam, F. Treussart, S. Turner, S. Bals, S. Pavel et al., Fluorescent nanodiamonds embedded in biocompatible translucent shells, Small 10, 1106 (2014).

[9] I. Rehor, H. Mackova, S. K. Filippov, J. Kucka, V. Proks, J. Slegerova, S. Turner, G. Van Tendeloo, M. Ledvina, M. Hruby et al., Fluorescent nanodiamonds with bioorthogonally reactive protein-resistant polymeric coatings, ChemPlusChem 79, 21 (2014).

[10] J. Havlik, V. Petrakova, I. Rehor, V. Petrak, M. Gulka, J. Stursa, J. Kucka, J. Ralis, T. Rendler, S.-Y. Lee et al., Boosting nanodiamond fluorescence: Towards development of brighter probes, Nanoscale 5, 3208 (2013).

[11] I. Kratochvílová, J. Sebera, P. Ashcheulov, M. Golan, M. Ledvina, J. Míva, F. Mravec, A. Kovalenko, D. Zverev, B. Yavkin et al., Magnetical and optical properties of nanodiamonds can be tuned by particles surface chemistry: Theoretical and experimental study, J. Phys. Chem. C 118, 25245 (2014).
[12] T. Fukui, Y. Doi, T. Miyazaki, Y. Miyamoto, H. Kato, T. Matsumoto, T. Makino, S. Yamasaki, R. Morimoto, N. Tokuda et al., Perfect selective alignment of nitrogenvacancy centers in diamond, Appl. Phys. Express 7, 055201 (2014).

[13] E. Van Oort, N. B. Manson, and M. Glasbeek, Optically detected spin coherence of the diamond NV centre in its triplet ground state, J. Phys. C 21, 4385 (1988).

[14] A. P. Nizovtsev, S. Ya. Kilin, C. Tietz, F. Jelezko, and J. Wrachtrup, Modelling fluorescence of single nitrogenvacancy defect centers in diamond, Physica (Amsterdam) 308B, 608 (2001).

[15] N. B. Manson, J. P. Harrison, and M. J. Sellars, Nitrogenvacancy center in diamond: Model of the electronic structure and associated dynamics, Phys. Rev. B 74, 104303 (2006).

[16] L. J. Rogers, S. Armstrong, M. J. Sellars, and N. B. Manson, Infrared emission of the NV centre in diamond: Zeeman and uniaxial stress studies, New J. Phys. 10, 103024 (2008).

[17] L. J. Rogers, M. W. Doherty, M. S. J. Barson, S. Onoda, T. Ohshima, and N.B. Manson, Singlet levels of the $\mathrm{NV}^{-}$ centre in diamond, New J. Phys. 17, 013048 (2015).

[18] Y. Dumeige, M. Chipaux, V. Jacques, F. Treussart, J.-F. Roch, T. Debuisschert, V. M. Acosta, A. Jarmola, K. Jensen, P. Kehayias et al., Magnetometry with nitrogen-vacancy ensembles in diamond based on infrared absorption in a doubly resonant optical cavity, Phys. Rev. B 87, 155202 (2013).

[19] L. Rondin, J. P. Tetienne, T. Hingant, J. F. Roch, P. Maletinsky, and V. Jacques, Magnetometry with nitrogenvacancy defects in diamond, Rep. Prog. Phys. 77, 056503 (2014).

[20] T. Wolf, P. Neumann, K. Nakamura, H. Sumiya, T. Ohshima, J. Isoya, and J. Wrachtrup, Subpicotesla Diamond Magnetometry, Phys. Rev. X 5, 041001 (2015).

[21] S. Steinert, F. Dolde, P. Neumann, A. Aird, B. Naydenov, G. Balasubramanian, F. Jelezko, and J. Wrachtrup, High sensitivity magnetic imaging using an array of spins in diamond, Rev. Sci. Instrum. 81, 043705 (2010).

[22] L. M. Pham, D. Le Sage, P. L. Stanwix, T. K. Yeung, D. Glenn, A. Trifonov, P. Cappellaro, P. R. Hemmer, M. D. Lukin, Hongkun Park et al., Magnetic field imaging with nitrogen-vacancy ensembles, New J. Phys. 13, 045021 (2011).

[23] D. Le Sage, K. Arai, D. R. Glenn, S. J. DeVience, L. M. Pham, L. Rahn-Lee, M. D. Lukin, A. Yacoby, A Komeili, and R. L. Walsworth, Optical magnetic imaging of living cells, Nature (London) 496, 486 (2013).

[24] V. Petráková, A. Taylor, I. Kratochvílová, F. Fendrych, J. Vacík, J. Kučka, J. Štursa, P. Cigler, M. Ledvina, A. Fišerová et al., Luminescence of nanodiamond driven by atomic functionalization: Towards novel detection principles, Adv. Funct. Mater. 22, 812 (2012).

[25] V. Petrakova, I. Rehor, J. Stursa, M. Ledvina, M. Nesladek, and P. Cigler, Charge-sensitive fluorescent nanosensors created from nanodiamonds, Nanoscale 7, 12307 (2015).

[26] K. Sokolov, Nanotechnology: Tiny thermometers used in living cells, Nature (London) 500, 36 (2013). 
[27] T. Plakhotnik, M. W. Doherty, J. H. Cole, R. Chapman, and N. B. Manson, All-optical thermometry and thermal properties of the optically detected spin resonances of the $\mathrm{NV}^{-}$-center in nanodiamond, Nano Lett. 14, 4989 (2014).

[28] L. P. McGuinness, L. T. Hall, A. Stacey, D. A. Simpson, C. D. Hill, J. H. Cole, K. Ganesan, B. C. Gibson, S. Prawer, P. Mulvaney et al., Ambient nanoscale sensing with single spins using quantum decoherence, New J. Phys. 15, 073042 (2013).

[29] J.-W. Zhou, P.-F. Wang, F.-Z. Shi, P. Huang, X. Kong, X.-K. Xu, Q. Zhang, Z.-X. Wang, X. Rong, and J.-F. Du, Quantum information processing and metrology with color centers in diamonds, Front. Phys. 9, 587 (2014).

[30] G. Balasubramanian, A. Lazariev, S. R. Arumugam, and D.-w. Duan, Nitrogen-vacancy color center in diamondemerging nanoscale applications in bioimaging and biosensing, Curr. Opin. Chem. Biol. 20, 69 (2014).

[31] I. Aharonovich, S. Castelletto, D. A. Simpson, C. H. Su, A. D. Greentree, and S. Prawer, Diamond-based singlephoton emitters, Rep. Prog. Phys. 74, 076501 (2011).

[32] T. A. Kennedy, F. T. Charnock, J. S. Colton, J. E. Butler, R. C. Linares, and P. J. Doering, Single-qubit operations with the nitrogen-vacancy center in diamond, Phys. Status Solidi (b) 233, 416 (2002).

[33] T. H. Taminiau, J. Cramer, T. van der Sar, V. V. Dobrovitski, and R. Hanson, Universal control and error correction in multi-qubit spin registers in diamond, Nat. Nanotechnol. 9, 171 (2014).

[34] L. T. Hall, G. C. G. Beart, E. A. Thomas, D. A. Simpson, L. P. McGuinness, J. H. Cole, J. H. Manton, R. E. Scholten, F. Jelezko, J. Wrachtrup et al., High spatial and temporal resolution wide-field imaging of neuron activity using quantum NV-diamond, Sci. Rep. 2, 401 (2012).

[35] J. F. Barry, M. J. Turner, J. M. Schloss, D. R. Glenn, Y. Song, M. D. Lukin, H. Park, and R. L. Walsworth, Optical magnetic detection of single-neuron action potentials using quantum defects in diamond, arXiv:1602.01056.

[36] O. Faklaris, D. Garrot, V. Joshi, F. Druon, J.-P. Boudou, T. Sauvage, P. Georges, P. A. Curmi, and F. Treussart, Detection of single photoluminescent diamond nanoparticles in cells and study of the internalization pathway, Small 4, 2236 (2008).

[37] R. Igarashi, Y. Yoshinari, H. Yokota, T. Sugi, F. Sugihara, K. Ikeda, H. Sumiya, S. Tsuji, I. Mori, H. Tochio et al., Real-time background-free selective imaging of fluorescent nanodiamonds in vivo, Nano Lett. 12, 5726 (2012).

[38] Y. Yoshinari, S. Mori, R. Igarashi, T. Sugi, H. Yokota, K. Ikeda, H. Sumiya, I. Mori, H. Tochio, Y. Harada et al., Optically detected magnetic resonance of nanodiamonds in vivo; implementation of selective imaging and fast sampling, J. Nanosci. Nanotechnol. 15, 1014 (2015).

[39] R. Chapman and T. Plakhoitnik, Background-free imaging of luminescent nanodiamonds using external magnetic field for contrast enhancement, Opt. Lett. 38, 1847 (2013).

[40] S. K. Sarkar, A. Bumb, X. Wu, K. A. Sochacki, P. Kellman, M. W. Brechbiel, and K. C. Neuman, Wide-field in vivo background free imaging by selective magnetic modulation of nanodiamond fluorescence, Biomed. Opt. Express 5, 1190 (2014).
[41] A. Hegyi and E. Yablonovitch, Molecular imaging by optically detected electron spin resonance of nitrogenvacancies in nanodiamonds, Nano Lett. 13, 1173 (2013).

[42] A simplified level scheme is used in the model (i) for the excited ${ }^{3} E$ multiplet which has a double total multiplicity and undergoes additional splitting and (ii) for the system of singlet states which in a recent report [18] were shown to consist of a lowest ${ }^{1} A$ state with a higher ${ }^{1} E$ level. However, for the considered optical cycle, the spin-selection rules are the essential element.

[43] J. P. Tetienne, L. Rondin, P. Spinicelli, M. Chipaux, T. Debuisschert, J. F. Roch, and V. Jacques, Magnetic-fielddependent photodynamics of single NV defects in diamond: An application to qualitative all-optical magnetic imaging, New J. Phys. 14, 103033 (2012).

[44] A. Jarmola, V. M. Acosta, K. Jensen, S. Chemerisov, and D. Budker, Temperature- and Magnetic-Field-Dependent Longitudinal Spin Relaxation in Nitrogen-Vacancy Ensembles in Diamond, Phys. Rev. Lett. 108, 197601 (2012).

[45] N. D. Lai, D. Zheng, F. Jelezko, F. Treussart, and J.-F. Roch, Influence of a static magnetic field on the photoluminescence of an ensemble of nitrogen-vacancy color centers in a diamond single-crystal, Appl. Phys. Lett. 95, 133101 (2009).

[46] A. Dréau, M. Lesik, L. Rondin, P. Spinicelli, O. Arcizet, J.-F. Roch, and V. Jacques, Avoiding power broadening in optically detected magnetic resonance of single NV defects for enhanced dc magnetic field sensitivity, Phys. Rev. B 84, 195204 (2011).

[47] R. Chapman and T. Plakhotnik, Anomalous saturation effects due to optical spin depolarization in nitrogenvacancy centers in diamond nanocrystals, Phys. Rev. B 86, 045204 (2012).

[48] G. Bogdan, M. Nesládek, J. D’Haen, J. Maes, V. V. Moshchalkov, K. Haenen, and M. D'Olieslaeger, Growth and characterization of near-atomically flat, thick homoepitaxial CVD diamond films, Phys. Status Solidi (a) 202, 2066 (2005).

[49] See Supplemental Material at http://link.aps.org/ supplemental/10.1103/PhysRevApplied.6.064013 for SM 1: ODMR spectra in SC and FNDs; SM 2: Magnetic field dependence of SMF contrast in SC; SM 3: Spin population relaxation time $\mathrm{T} 1$ in the ground triplet; SM 4: Calculation of contrast excitation rate dependence for high Rabi frequency; SM 5: Slice illustrative for FND selectivity and defocusing series in DIC and SMF images; SM 6: SMF contrast in stained samples in low vs. high magnification; SM 7: Depth slices of FNDs fluorescence in confocal microscopy; SM 8: 3D representation of the FNDs distribution in the cell culture; SM 9: Micrograph of CVD grown layer and confocal PL for NV concentration profile in SC.

[50] E. Bourgeois, A. Jarmola, P Siyushev, M. Gulka, J. Hruby, F. Jelezko, D. Budker, and M. Nesladek, Photoelectric detection of electron spin resonance of nitrogen-vacancy centres in diamond, Nat. Commun. 6, 8577 (2015).

[51] J. Stursa, J. Havlik, V. Petrakova, M. Gulka, J. Ralis, V. Zach, Z. Pulec, V. Stepan, S. A. Zargaleh, M. Ledvina et al., Mass production of fluorescent nanodiamonds with a narrow emission intensity distribution, Carbon 96, 812 (2016). 
[52] T. G. Ruardij, M. H. Goedbloed, and W. L. C. Rutten, Adhesion and patterning of cortical neurons on polyethylenimineand fluorocarbon-coated surfaces, IEEE Trans. Biomed. Eng. 47, 1593 (2000).

[53] M. Mahmud, R. Pulizzi, E. Vasilaki, and M. Giugliano, QSpike tools: A generic framework for parallel batch preprocessing of extracellular neuronal signals recorded by substrate microelectrode arrays, Front. Neuroinform. 8, 26 (2014).

[54] V. M. Acosta, E. Bauch, M. P. Ledbetter, C. Santori, K.-M. C. Fu, P. E. Barclay, R. G. Beausoleil, H. Linget, J. F. Roch, F. Treussart et al., Diamonds with a high density of nitrogen-vacancy centers for magnetometry applications, Phys. Rev. B 80, 115202 (2009).
[55] A. P. Hopper, J. M. Dugan, A. A. Gill, O. J. L. Fox, P. W. May, J. W. Haycock, and F. Claeyssens, Amine functionalized nanodiamond promotes cellular adhesion, proliferation and neurite outgrowth, Biomedical materials $\mathbf{9}$, 045009 (2014).

[56] Y.-A. Huang, C.-W. Kao, K.-K. Liu, H.-S. Huang, M.-H. Chiang, C.-R. Soo, H.-C. Chang, T.-W. Chiu, J.-I. Chao, and E. Hwang, The effect of fluorescent nanodiamonds on neuronal survival and morphogenesis, Sci. Rep. 4, 6919 (2014).

[57] D. Maysinger, J. Ji, E. Hutter, and E. Cooper, Nanoparticlebased and bioengineered probes and sensors to detect physiological and pathological biomarkers in neural cells, Front. Neurosci. 9, 480 (2015). 